a N. Cooper, d'après ma demande expresse, m'écrivait le 3 Juin $1 \times 34$ que l'ouverture libre étail 13,3 el Je foyer 25 pieds 3 ponces, mesures anglaises $\left.{ }^{*}\right)=12 \frac{1}{3}$ pouces et 23 pieds 9 pouces, mesures de france. A peine cet objectif fut-il nonté cn bois que M. Cooper en parut très-satisfait. Mais, dans sa iellre du 3 Juin, après mavoir annoncé la terminaison du tube en fer, il ajoute, "le champ de vue est beaucoup plus foncé," et il m'envoye plusieurs copies de la description de l'equatorial, avec une carte céleste manuscrite contenant ses découverles. 1'où je puis conclure que $M$. Cooper, avec me munlure très-solide, obtenant succesivement de plus beaux effets par les soins qu'il apporte au centrage de son objectif, fournit la meilleure preuve de la perfection que jai pu donner anx lentilles dont il se compose.

L'excellente lunette (comme il le dit lui-mêne) avec laquelle M. Dumouchel a vu le premier Ja comète fut envoyée par moi ì liome en 1829 , pen après la fourniture que ie fis d'un obieclif semblable pour l'instrument des passages placé à l'observatoire Hoyal de Paris.

Depuis que ma mauvaise santé m'a forcé de céder mon etablissement î M. Rossin, mon neveu, le travail des grands objectif ne s'est pas arrèté. Il a déji torminé un télescope de $8 \$$ pouces d'ouverture et de 12 pieds de foyer. Il s'occupe cn ce monent d'objectifs de 13 pouces de diamètre. Jai lieu de penser que les moyens par lesquels j'ai réussi

*) Au reste c'est ausi qu'en parlent toutes les relations anglaises. lui donneront les mêmes succès el je suis persuadé qu'il terminerait tout aussi heureusement des entreprises phis considérables.

En comparant l'élablissement de Munich à celui que j'ai formé péniblement à Paris, les savaus devraient peut-être considérer les circonstances particulieres à l'un et à l'autre. On vánte, on préconise les ateliers, les instrumens gigantes. ques de Munich, et mon nome niest plus même prononcé quand on parle des télescope sortis de mes mains, dont trois cépendant dépassent tout ce qu'a produit Munich, où les fonds ont été versés largement, et des commandes importantes ont été faites, mais chez moi, toul ce qui a élé exécuté, je l'ai entrepris à mes risques. Un premier objectif de $8 \frac{1}{4}$ pouces; les deux objectifs de 11 pouces, celui de $12 \frac{1}{2}$ pouces, plusieurs de 5,6 et 7 pouces, tout a élé commencé ef terminé sans commande aucune et tout a úté livré après des épreuves multipliées au gré des acquéreurs, souvent chez eux, et prolongées autant qu'ils ont pu le désirer.

Mon neveu peut offrir les mêmes avanlages dont l'importance est facile à saisir: car c'est une position bien différente de commander un instrument dont la réussite plus ou moins heureuse peut faire nailre quelques regrets et donner lieu à des débats désagréables, ou d'acheter celui qu'on a pu soumettre à toute sorte d'épreuves.

J'ai l'honneur d'être

Le 3 Mars 1836 à Deuil, près de

Montmorency, aux environs Clauchoix. de Paris.

\title{
Observations faites dans la Mer des Antilles.
}

Par Mr. Zahrtmann, Capitaine de Haut Bord, Directeur du Depôt Royal des Cartes de la Marine.

Pendant mon scjour aux Antilles en 1833-34 j'ai continué les Iravaux commencés en 1825-26 dont jai douné les détails dans le Nr. 113 de ce jourual.

Jai placé una lunelle méridienue dans un observatoire qui vient d'être établi par Mr. Kier, Aide Capilaine dı port de St. Thomas, dans la vicille tour des Flibustiens qui fomine la ville, el qui se liouve placée dans la méridienne du Vont Christian. Cel observatoire avait plusienrs avantages

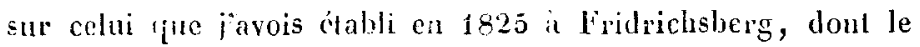
plus escentiel ent ceini dere visible de lobservatoire de Mr. Lan:r dans rìle de St. Croix. Par une opération géodésique ie lidi krouvé placé $14^{\prime \prime} 6$ au nord, et $1{ }^{\prime \prime} 25 \mathrm{en}$ tems à l'ouest de mon ancien observaloire.

Les observaiious suivantes onl été faites pour en déterminer la position
Hauteurs Circomméridiennes.

1833 Oct. 25. 14 Observations donnent la latitude $18^{\circ} 20^{\prime} 31^{\prime \prime} 5$

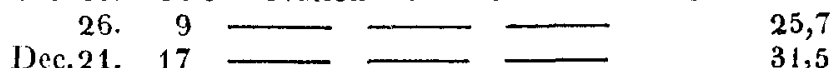

1834 Janv.11. $11 \div \square-32,4$ lévr. 3. 5 - $-\square$

13. $22-50,7$

13. $24-\square 34,6$

15. $19-\longrightarrow 23,7$

par 121 Observations, la latitude $\overline{18^{\circ} 20^{\prime} 34^{\prime \prime} 5}$

Latilude de Fridrichsberg $1834 \frac{-14,6}{-18^{\circ} 20^{\prime} 20^{\prime \prime} 0}$

en $1826-18 \quad 20 \quad 23,0$

Lobzervatoire de Mr. Lang est en $17^{\circ} 44^{\prime} 32^{\prime \prime}$ de latilude et d'après les plus récentes calculs de Mr. Wurm (Astr. Nachr. Nr. 200) à $4^{\text {h }} 28^{\prime} 4^{\prime \prime} j$ à louest de Paris. Mr. $\operatorname{Lang}$ y a ob- 
servé Y'Azimuth de l'observaloire de St 'Thomas N. $21^{\circ} 4^{\prime} 9^{\prime \prime} 5$ ouest, ce qui donne pour la différnce en longilude des deux observaloires...................... $58^{\prime \prime 24}$

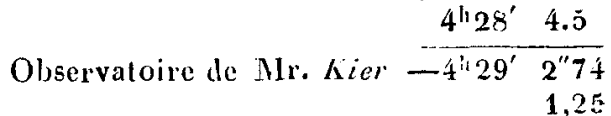

Cidevant observatoire de Findruhberg $\overline{4^{\text {ln } 29^{\prime}}}$ 1"49 $^{\prime \prime}$

Les chronomètres en 1834 (en accord avec te resultat qu'ils donuaient en 1826) firent cetle difference plus petile, savoir:

\begin{tabular}{|c|c|c|c|c|c|c|}
\hline \multirow{3}{*}{1833} & \multirow{2}{*}{\multicolumn{2}{|c|}{ Inillut an }} & \multirow{2}{*}{$\begin{array}{c}A \\
58^{\prime \prime} 34\end{array}$} & \multirow{2}{*}{$\begin{array}{c}B \\
59^{\prime \prime} 40\end{array}$} & $C$ & \multirow{2}{*}{$\underset{59^{\prime \prime} 42}{D}$} \\
\hline & & & & & & \\
\hline & Août & 9. & 56,39 & 55,88 & & 55,84 \\
\hline & & 14. & 58,07 & 57,48 & & 56,25 \\
\hline & Octobr & e 6. & 57,61 & 57,00 & & 56,24 \\
\hline & & 10. & 57,34 & 57,01 & & 55,87 \\
\hline & & 30. & 56,87 & 57,012 & & \\
\hline & Déc. & 17. & 56,43 & 56,04 & $56 " 17$ & 57,05 \\
\hline 1834 & $\begin{array}{r}\text { Févr. } \\
\qquad \begin{array}{c}A \\
C .\end{array}\end{array}$ & 20. & , & $\begin{array}{c}56,4 i \\
B \ldots \\
D \ldots B\end{array}$ & $\begin{array}{l}56,69 \\
4.31258 \\
\text { ucd. }\end{array}$ & 58,51 \\
\hline
\end{tabular}

Comme les comparaicons avec la pendule de lobservaloire de Mr. Laug ont loujours élé failes par signaux, a des dislances assez cousilimables, il fant resinter ce résullat comme élant d'un moindre poids que celui tscé d'un aztmuth observé directement, et de deux latiludes bien délerminées. Je commencerai donc par corriger les longitudes que j'ai determinées en 1826 hasées sur la posilion de loolservatoire de Fridrichsberg comme point de depart:

$$
\begin{aligned}
& 4^{\text {h }} 29^{\prime} 1^{\prime \prime} 49 \text { longilude de Fridrichsberg a St. Thomas. } \\
& \text { - } 9,174^{\text {li } 28^{\prime} 52^{\prime \prime} 32} \text { St. Croix, liridrichsforl } \\
& 30,72 \quad 2830,72 \text { St. Jean, Crul Bai } \\
& \text { - } 9^{\prime} 10,01 \quad 1951,48 \text { Nieves, Charleston } \\
& \text { - } 1459,36 \quad 14 \quad 2,13 \text { la Martinique, St. Pierre } \\
& \text { - } 1524,77 \quad 1336,72 \text { ldem forl hoyal } \\
& +1220,174121,66 \text { Venezuela, P'uerto Cabello } \\
& 4^{\text {h }} 28^{\prime} 4^{\prime \prime} 5 \text { longilude de Si. Croix } \\
& 849,5 \text { (Ni. } 90 \text { Astr. Nachr.) } \\
& \text { 4h36'54"0 Castillo de Mulatos } \\
& 10,0 \text { Reduct. à la Guayra } \\
& 4^{\mathrm{h}} 37^{\prime} 4^{\prime \prime} 0 \text { longitude de la Guayra. }
\end{aligned}
$$

La différence en longilule entre lubservatoire de Mr. Kier. et le forl Bocca del lío de Cumana a éte trouvéc:

Par le chronometre $\left.\begin{array}{r}A-2^{\prime} 58^{\prime \prime} 10 \\ B-256,95\end{array}\right\}$ Noyent $-2^{\prime} 57^{\prime \prime} 53$

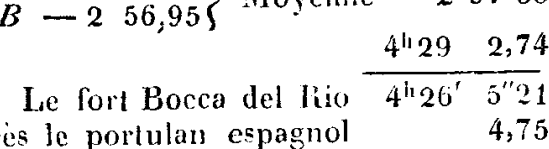
réduction dapres le portulan espagnol

Le forl San Antonio de Cumanit $\overline{4^{1 / 2}-6^{\prime} 0^{\prime \prime} 46}$

Le furt Amsterdian is Curacio

a l'onest de l'uerto Cabello par le Chronometre $A$ 3'41"05 $B \quad 342,01$

Moyenme $3^{\prime} 41^{\prime \prime} 53$

Longitude de Puerto Cabello $1826-\frac{4^{\text {h }} 4121,66}{4^{\text {h }} 45^{\prime} 3^{\prime \prime} 19}$ à l'onest du fort Bocca del Rico par le Chronomètre $A$ th $0^{\text {ht }} 19^{\prime} 0^{\prime \prime} 06$

B $019 \quad 1,11$

Longilude du fort Bocca del Rio $\begin{array}{ccc}4 & 26 & 5,21\end{array}$

$4^{\text {h }} 45^{\prime} \quad 5^{\prime \prime} 80$

$4.45 \quad 3,19$

Par moyenue longitude du fort Amsterdam à Curaçao $4^{\text {h }} 45^{\prime} 4^{\prime} 50$

$4^{\text {h } 29^{\prime}} 2^{\prime \prime} 74$ Observatoire de St Thomas.

- $853,794^{\text {hr } 20^{\prime}} 8^{\prime \prime} 95$ Sl. Christophe, Basseterre fort Smith......... 17 $17^{\prime} 45^{\prime \prime} 0$

-12 12,67 16 50,07 Antigue, St. Johns, fort

James.............. $17 \quad 8 \quad 0,0$

-12 39,80 16 22,94 la(iuadeloupe, Basseterre, fort de Thois......... 16 o 30,3

$-1526,15 \quad 13 \quad 36,59$ la Martumque, le forthoyal

$+855,43 \quad 3758,17$ Piertoricco, Mayaguez, Batteric la Puntilla

+ 649,4335 52,17 Idem, Ponce, Ballerie de la Playa......... 175721,0

A lexception de la latitude de Ponce, déduite d'une serie de hauteurs circomméridienmes observée a bord el sur l'horizon naturel daus les circorstances les plus fovorables, lontes les autres latitudes rósultent de hauteurs circomméridiennes observées à terre sur un horizon artificicl. Les longitules ont élé tronvées en délerminant le midi vrai par des Lrauteurs correspondantes, excepté à St. Jean d'Antigue où îl n'y a pas eu de correspondantes l'après midi el où on a élé obligé de calculer le temps vrai sur les hanteurs absolues du matin en $y$ employant la latitule trouvée par les hauteur's circomméridiennes. L'exactitude de ces délerminations me parait garanti par l'accord satisfaisant qui se trouve dans les deux délerminations du forl Amsterdam à Curacao, par Cumana el par P'uerto Cabello, ainsi que par le parfait accord entre les deux déterminalions du Fort-lioyal, en 1826 et 1834 .

Les observations suivantes ont élé faites à bord ell passant pries des points en question:

Blanchille, pointe S. O. $4^{\mathrm{h}} 27^{\prime} 49^{\prime \prime} 94$

Tortugas, poinleE. 43011,88 incertaine par cause de

Cap Codera, pointe S. E. 43334,0 (la distance.

Centincla 43338,9 et is $N 12^{\mathrm{h}} 22^{\prime} 20^{\prime \prime} \mathrm{O}$ du monde de la pointe li de CapCodera; lal. $10^{\circ} 52^{\prime} 20^{\prime \prime}$.

Orcbilla, pointe O. $\quad 43417,7$ et la lalitule $11^{\circ} 50^{\prime} 12^{\prime \prime}$ (très-précise).

Un liazard malencontreux m'a fait perdre loccasion de Lier la Ville St. Juan de Puerturicco directement avec Jobservatoire da St. 'Thomas, mais comme je suppose que celle longilude differe essentiellenent de celle adopté génciralement je vats communiquer celle qui me parait la plus probable:

St. Thomas, St.Juan de Pueriorico selonChurruca $0^{\circ} 4^{\prime} 44^{\prime \prime} 73$,

Moyenne $\frac{42,80}{0^{\mathrm{h}} 4^{\prime} 43^{\prime \prime} 77}$

Lougilude de St. Thomals $429 \quad 2,74$ 
La'lrinité-Puertoriccoselon Churruca $0^{\text {h }} 18^{\prime} 21^{\prime \prime} 0$

Là'Trimlé(A.N. Nr.221 Capt. Seidelin) $415 \quad 23,70$

$$
\frac{1}{4^{\mathrm{i}} 33^{\prime} 44^{\prime \prime} 70}
$$

St. Juan de Ulloa - Aguadilla par Luyando

5. Juan de Puertorirco - Aguadilla (Ferrer,

Conu. des tems 1817)................

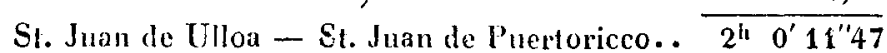

St. Juan de Ulloa — el Morro de Havana...... 0 o 55 4,00

el Morro de Havana - el Morro de Puertoricco 1h $^{\text {h }} 5^{\prime \prime} 47$

Longilude du Morro de Havana.......... 5 38 50,8

I.ongitude du Morro de St. Juan de Puertoricco par St. Thomas $4^{\mathrm{h}} 33^{\prime} 46^{\prime \prime} 51$ par Trinidad 44,70 par la Havana et Veracruz

44,70
43,33

par Cerquero, Calcul de l'occultation de $\alpha$ Tauri par

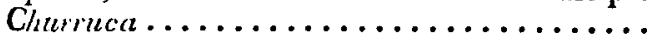

par Ferrer, distances lunaires, calculées par lui

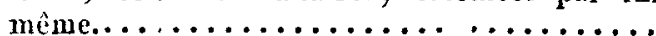

Moyenne $\overline{4^{\mathrm{h}} 33^{\prime}} 45^{\prime \prime} 10$

11 me parail que laccord qui existe entre ces différentes déterminations, garanti l'exactilude de la longitude acloptée pour ce point impurlant.

I.'inclinaison de l'Aiguille fut trouvé:

$$
\text { à Curaego. }
$$

1833 le 11 Sept. $38^{\circ} 27^{\prime}$

$$
\begin{aligned}
& 12-3838 \\
& 14-\quad 3836 \\
& 21-\quad 3854
\end{aligned}
$$

1833 le 26 d'Othonias.

$49^{\circ} 8^{\prime}$

$1834 \quad 14$ Février 4950

J'ai à regreller que les observations sur lintensilé de la force magnétique que j'ai fait faice en grand nombre, ont élé inutiles parceque láaguille avoil perdue considérablement de sa force.

L'aignille après avoir fait

a Copenhague le 27 d'Avril 300 oscillationsen $1212^{\prime \prime} 03$

fit à Curaçao le 16 de Sepl. 300 oscillations en 856,52

$$
\begin{aligned}
& 17 \text { — } \quad \text { - } \quad \text { - } \\
& 19-\quad \text { - } \quad 857,92
\end{aligned}
$$

à St. Thomas le 22 d'Oclobre - $\quad 900,33$

De retour à Copenhague l'aiguille n'y lit le 17 de Nai 1834 fue 300 oscillat. en $1325^{\prime \prime} 59$, el le 17 Déc.' 1835 en $1308^{\prime \prime} 52$.

Des relivemens pris par Mr. Lang à St. Croix, de différens points visibles, ont servi à en chéterminer la longitude par moyen des latitudes déjà connues. Ces points sont, entre l'observatoire de St. Thomas:

Puertorico, Sierra de Luquillo $43242^{\prime \prime}, 918^{\circ} 19^{\prime} 12^{\prime \prime}$ Churruca St. Thomas, la Caravelle (Sail

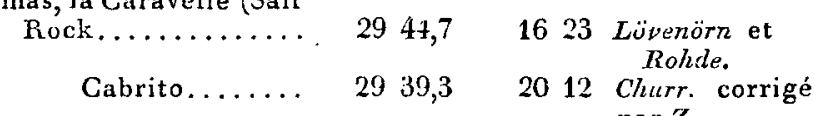

Cape d'Oisean.. $2844,1 \quad 1350$ Rohde corr.p.Z.

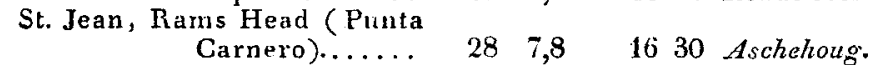

Flanagan (C.Cenoejos) $275 \%, 8050$ Churruca.

Virgin Gorda, Cap Pajaro.... $26 \quad 36,9 \quad 3040$ Chur. et Lüven.

Il faut convenir que cetle Iongitude de la Siera de Liquillo ne correspond point avec celle que j'ai adoptée pour la ville de St. Jean, en prenant ces denx points daus la carte de Churruca. Aussi doit elle êlre considérablement affectée par toute erreur dans la latitude, et celles de Churruca paraissent the trop fortes dans celle partie des Anlilles. C'e:t ainsi qu'il trouvầt pour la katilude du fort Christian à St. Thomas $18^{\circ} 21^{\prime} 16^{\prime \prime}$; la position du bâton de pavillon est: 57,1 pieds tanois à l'ouest et 1132,2 pieds an sud de l'observat. de Mr. Lier, donc en $18^{\circ} 20^{\prime} 23^{\prime \prime}$ de latilude.

Dans presque toutes les cartes, surlout les cartes anglaises, l'ille de Nièves a élé placée troj au nord. Un amateur d'astronomie, le juge, Mr. Webb, y Irouva la latitude de son observatoire à Sturey Hill: $17^{\circ} 8^{\prime} 47^{\prime \prime}$.

Dans la campagne de Mr. Bolenhoff avec le brig St. Jean, on détermina la longitude du batton le pavillon ä Riossan dans l'île de Dominique à $4^{\mathrm{h}} 15^{\prime} 0^{\prime \prime} 20$, partant de l'observatoire de Mr. Lang. La position de lilôt Áves a élé delerminée:

par Mr. Ulldall Capilaine de Vaisseau à $15^{\circ} 40^{\prime} 36^{\prime \prime}$ el $4^{\mathrm{l}} 24^{\prime} 0^{\prime \prime} 5$

Sir Thomas Cuchrane Idem à 154030 el $424 \quad 1,6$ Il me parail remarquable que la longilude de la Trinité et de la Guayra, determinées par Mr. Seiclelin el moi, s'accoirlent à indiquer qu'il-y-aura $9 \mathrm{~s} 3$ à ajouter aux longitudes de Mr. Forsler, pour en tirer la longitude posilivede ces deux lieux; quant à la position rélative des trois points: St. Croix, la Trinité et la Guayra il n'y.pourra plus exister d'incerlitude, vu le parfait accord entre nous 3 observateurs. C'est très-curieux que celle même différence de $9^{s} 3$ se retrouve dans la longitude de Para le dernier point au Brésil déterminé par Mr. F'orster, quand on la compare à celle tronvée daus l'expédition de Mr. Roussin. Ceci prouve que pour le Brésil il faut préférer les longitudes de Mr. Givry, et puis que l'examen de lexacte longitude de l'observaloire de Mr. Lang, déduite de toules les observations qui y ont élé failes, seroil un travail du plus grand inlerêt non seulement pour la géographie des Antilles, mais encore pour celle de tont l'Amériques:

Fieste à Vouf communiquer que la plupart des observations de ma deqnière campagne a dé faite par Mr. Schultz, lieutenant de gate qui à beaucoup d'application rémit tontes les antres qualités d'un excellent observateur.

Zahrtmanu.

Ueber die Wicderkehr des Pons'schen (Enckeschen) Cometen im Jahre 183j und die Hypothese des widerstehenden Mittels. Von Herrn Prof. und Ritter Encke (Beschlu[s). p. 265. - Schreiben des, Herrn Cauchorx an den Herausgeber. p 273. - Observations faites dans la Mer des Antilles. Par Mr. Zahrtmann, Capitane de Haut Bord etc. p. 275. 